\title{
TWO RADIUS OF CONVEXITY PROBLEMS
}

\author{
CARL P. MCCARTY
}

ABSTRACT. The sharp radius of convexity of functions with prescribed second coefficient is found for the two classes: functions starlike of order $\alpha$, and functions whose derivative has real part greater than $\alpha$.

1. Introduction. Let $\mathscr{P}(\alpha)$ denote the class of functions $P(z)=$ $1+b_{1} z+\cdots$ which are analytic and satisfy $\operatorname{Re}\{P(z)\}>\alpha$ for $|z|<1$ where $\alpha \in[0,1)$. If $\varepsilon=\exp \left\{-i \arg b_{1}\right\}$ then $P(\varepsilon z)=1+\left|b_{1}\right| z+\cdots$ and we see that it is no actual restriction to limit our study of $\mathscr{P}(\alpha)$ to functions with a nonnegative real first coefficient. It is known [2] that $\left|b_{1}\right| \leqq 2(1-\alpha)$ and we define $\mathscr{P}_{b}(\alpha)=\left\{P(z) \in \mathscr{P}(\alpha): P^{\prime}(0)=2 b(1-\alpha)\right\}$ for $b \in[0,1]$. This paper extends results found in [1] by obtaining a lower bound on $\operatorname{Re}\left\{z P^{\prime}(z) / P(z)\right\}$ for $P(z) \in \mathscr{P}_{b}(\alpha)$ and subsequently applying the results to obtain a sharp estimate for the radius of convexity of the two classes $\mathscr{S}_{a}^{*}(\alpha)$ and $\mathscr{P}_{a}^{\prime}(\alpha)$ for each $a \in[0,1]$ and $\alpha \in[0,1)$ where

$$
\mathscr{P}_{a}^{\prime}(\alpha)=\left\{F(z)=z+a(1-\alpha) z^{2}+\cdots: F^{\prime}(z) \in \mathscr{P}_{a}(\alpha)\right\}
$$

and

$$
\begin{aligned}
\mathscr{S}_{a}^{*}(\alpha)=\left\{f(z)=z+2 a(1-\alpha) z^{2}\right. & +\cdots: \\
& \left.\operatorname{Re}\left\{z f^{\prime}(z) / f(z)\right\}>\alpha \text { for }|z|<1\right\} .
\end{aligned}
$$

The technique used to obtain the results is based on a method of Singh and Goel [4] and extends some of the results found therein.

2. Preliminaries. Let $\mathscr{A}$ denote the class of functions $w(z)$ such that $w(0)=0$ which are also analytic and satisfy $|w(z)|<1$ for $|z|<1$. We will occasionally use $\beta=2 \alpha-1$ to simplify computations and statements of results.

Received by the editors December 22, 1972 and, in revised form, March 23, 1973. AMS (MOS) subject classifications (1970). Primary 30A32, 30A76; Secondary $30 \mathrm{A04}$.

Key words and phrases. Radius of convexity, functions starlike of order $\alpha$, functions with positive real part.

(c) American Mathematical Society 1974 
LEMma 1. If $P(z) \in \mathscr{P}_{b}(\alpha)$, then $\left|P(z)-A_{b}\right| \leqq D_{b}$ for $b \in[0,1]$ where

$$
\begin{aligned}
A_{b}=\frac{(1+b r)^{2}-\beta(b+r)^{2} r^{2}}{\left(1+2 b r+r^{2}\right)\left(1-r^{2}\right)}, \quad D_{b} & =\frac{(1-\beta)(b+r)(1+b r) r}{\left(1+2 b r+r^{2}\right)\left(1-r^{2}\right)}, \\
\beta=2 \alpha-1 & \text { and } \quad r=|z|<1 .
\end{aligned}
$$

Proof. It is known that $P(z) \in \mathscr{P}_{b}(\alpha)$ if and only if there exists some $w(z) \in \mathscr{A}$ such that

$$
P(z)=[1+(1-2 \alpha) w(z)] /[1-w(z)] .
$$

So $w(z)=[P(z)-1] /[P(z)+(1-2 \alpha)]=b z+\cdots=z \varphi(z)$ where $\varphi(z)$ is analytic and $|\varphi(z)| \leqq 1$ for $|z|<1$ with $\varphi(0)=b$. Now, $(\varphi(z)-b) /(1-b \varphi(z))<$ $z$ and it follows that $\varphi(z)<(z+b) /(1+b z)$ and

$$
|w(z)|=|z \varphi(z)| \leqq|z|(|z|+b) /(1+b|z|) .
$$

Let

$$
g(z)=\frac{|z|+b}{1+b|z|} z \text { and } T(z)=\frac{1+(1-2 \alpha) z}{1-z} .
$$

We note that the image of $|z| \leqq r$ under $g(z)$ is a disk and that $T(z)$ is a bilinear transformation. The image of $|z| \leqq r<1$ under $P(z)$ is contained within the image of $|z| \leqq r$ under $(T \circ g)(z)$ which proves the lemma.

Remark 1. For a fixed $r \in[0,1), A_{b}-D_{b}$ decreases as $b$ increases over the interval $[0,1]$ since

$$
\frac{\partial}{\partial b}\left(A_{b}-D_{b}\right)=-\frac{(1-\alpha)\left(1-r^{2}\right)}{\left(1+2 b r+r^{2}\right)^{2}}<0 .
$$

LEMMA 2. Let $P(z) \in \mathscr{P}_{b}(\alpha), \beta=2 \alpha-1 \in[-1,1)$, and $k \geqq 1$; then for $b \in[0,1]$

$$
\begin{array}{r}
\operatorname{Re}\left\{k P(z)+\frac{\beta}{P(z)}\right\}-\frac{|z|^{2}|P(z)-\beta|^{2}-|P(z)-1|^{2}}{\left(1-|z|^{2}\right)|P(z)|} \\
(k+\beta)+2((k+2) \beta+k) b r \\
+\left((k+1)(\beta+1)^{2} b^{2}+2(k+\beta)-(\beta-1)^{2}\right) r^{2} \\
\geqq \frac{+2(k \beta+(k+2)) \beta b r^{3}+(1+k \beta) \beta r^{4}}{\left(1+2 b r+r^{2}\right)\left(1+(\beta+1) b r+\beta r^{2}\right)}
\end{array}
$$

if $R_{b} \geqq R^{\prime}$

(3) $\geqq 2\left((1+k)(1+\beta) A_{1}\right)^{1 / 2}-A_{1}$ if $R_{b} \leqq R^{\prime}$

where $A_{b}$ and $D_{b}$ are as in Lemma 1 with $R_{b}=A_{b}-D_{b}$ and

$$
R^{\prime}=\left((1+\beta) A_{1} /(1+k)\right)^{1 / 2} \text {. }
$$


Proof. Let $P(z)=A_{b}+u+i v$ and $R^{2}=\left(A_{b}+u\right)^{2}+v^{2}$ with $r=|z|$, then

$$
\begin{aligned}
\operatorname{Re}\{k P(z)+ & \left.\frac{\beta}{P(z)}\right\}-\frac{|z|^{2}|P(z)-\beta|^{2}-|P(z)-1|^{2}}{\left(1-|z|^{2}\right)|P(z)|} \\
= & \left(k-2\left(\frac{1-\beta r^{2}}{1-r^{2}}\right) R^{-1}+\beta R^{-2}\right)\left(A_{b}+u\right) \\
& +R+\left(\frac{1-\beta^{2} r^{2}}{1-r^{2}}\right) R^{-1} .
\end{aligned}
$$

Since $A_{1}=\left(1-\beta r^{2}\right) /\left(1-r^{2}\right)$ and $D_{1}=((1-\beta) r) /\left(1-r^{2}\right)$, the right-hand side of (4) may be written

$$
\left(k-2 A_{1} R^{-1}+\beta R^{-2}\right)\left(A_{b}+u\right)+R+\left(A_{1}^{2}-D_{1}^{2}\right) R^{-1} .
$$

Let $S_{b}(u, v)$ denote the expression appearing in (5), then regrouping the terms we have

$$
\begin{aligned}
S_{b}(u, v) & =\left(k+\beta R^{-2}\right)\left(A_{b}+u\right)+\left(\left(\left(A_{b}+u\right)-A_{1}\right)^{2}+v^{2}-D_{1}^{2}\right) R^{-1} \\
\partial S_{b} / \partial v & =v R^{-4} T_{b}(u, v) \\
T_{b}(u, v) & =-2 \beta\left(A_{b}+u\right)+\left(D_{1}^{2}-\left(A_{b}+u-A_{1}\right)^{2}-v^{2}\right) R+2 R^{3} \\
& =-2 \beta\left(A_{b}+u\right)+\left(D_{1}^{2}+2 A_{1}\left(A_{b}+u\right)-A_{1}^{2}\right) R+R^{3}
\end{aligned}
$$

Denote by $F_{b}(R)$ the right-hand side of (7) with $R \cos \psi=A_{b}+u$; then

$$
F_{b}(R)=2\left(A_{1} R-\beta\right) R \cos \psi+\left(D_{1}^{2}-A_{1}^{2}+R^{2}\right) R .
$$

Geometrical considerations show that for $R \in\left[A_{b}-D_{b}, A_{b}+D_{b}\right]$ the function $F_{b}(R)$ increases with increasing $R$. Since $R \cos \psi$ is the projection onto the real axis, it must lie on the diameter of the circle of Lemma 1; we have $R \cos \psi \geqq A_{1}-D_{1}$ by virtue of Remark 1 and so for all $b \in[0,1]$

$$
\begin{aligned}
F_{b}(R) & \geqq\left(2\left(A_{1}\left(A_{1}-D_{1}\right)-\beta\right)+\left(D_{1}^{2}-A_{1}^{2}+\left(A_{1}-D_{1}\right)^{2}\right)\right)\left(A_{1}-D_{1}\right) \\
& =\left((1-\beta)\left(\frac{1-\beta r^{2}}{(1+r)^{2}}\right)\right)\left(A_{1}-D_{1}\right)>0 .
\end{aligned}
$$

Hence the minimum of $S_{b}(u, v)$ inside the circle $\left|P(z)-A_{b}\right| \leqq D_{b}$ is attained on the diameter. Setting $v=0$ in (5) we obtain

$$
L_{b}(R)=(1+k) R+(\beta+1) A_{1} R^{-1}-2 A_{1}
$$

where $R=A_{b}+u \in\left[A_{b}-D_{b}, A_{b}+D_{b}\right]$. The absolute minimum of $L_{b}(R)$ in $(0, \infty)$ is attained at

$$
R^{\prime}=\left((1+\beta) A_{1} /(1+k)\right)^{1 / 2}
$$


and yields (3). Clearly $R^{\prime}<A_{b}+D_{b}$, but it is not always true that $R^{\prime} \in$ $\left[A_{b}-D_{b}, A_{b}+D_{b}\right.$ ]. When $R^{\prime}$ is not in the interval then $L_{b}(R)$ achieves its minimum at the point $R_{b}=A_{b}-D_{b}$ from which we get (2). The transition from (2) to (3) takes place for those values of $k$ and $\beta$ for which $R^{\prime}=R_{b}$.

LEMMA 3 [4]. If $w(z) \in \mathscr{A}$, then for $|z|<1$

$$
\left|z w^{\prime}(z)-w(z)\right| \leqq \frac{|z|^{2}-|w(z)|^{2}}{1-|z|^{2}} .
$$

TheOREM 1. Suppose $P(z) \in \mathscr{P}_{b}(\alpha)$ for $\alpha \in[0,1)$, then, for all $b \in[0,1]$,

$$
\begin{aligned}
\operatorname{Re}\left\{\frac{z P^{\prime}(z)}{P(z)}\right\} & \geqq \frac{-2(1-\alpha) r}{1+2 \alpha b r+(2 \alpha-1) r^{2}} \frac{b+2 r+b r^{2}}{1+2 b r+r^{2}} & \text { if } R^{\prime} \leqq R_{b} \\
& \geqq\left(2\left(\alpha A_{1}\right)^{1 / 2}-A_{1}-\alpha\right) /(1-\alpha) & \text { if } R^{\prime} \geqq R_{b}
\end{aligned}
$$

where $R_{b}=A_{b}-D_{b}, R^{\prime}=\left(\alpha A_{1}\right)^{1 / 2}$, and $r=|z|<1 ; A_{b}, D_{b}$ as in Lemma 1 .

Proof. Taking the logarithmic derivative of both sides of (1) we get

$$
\begin{aligned}
& \operatorname{Re}\left\{\frac{z P^{\prime}(z)}{P(z)}\right\}=(1-\beta) \operatorname{Re}\left\{\frac{z w^{\prime}(z)}{(1-w(z))(1-\beta w(z))}\right\} \\
& \geqq(1-\beta)\left(\operatorname{Re}\left\{\frac{w(z)}{(1-w(z))(1-\beta w(z))}\right\}\right. \\
&\left.\quad-\left|\frac{z w^{\prime}(z)-w(z)}{(1-w(z))(1-\beta w(z))}\right|\right) \\
&(1-\beta)^{-1}\left(\operatorname{Re}\left\{P(z)+\frac{\beta}{P(z)}-(1+\beta)\right\}\right. \\
&\left.-\frac{|z|^{2}|P(z)-\beta|^{2}-|P(z)-1|^{2}}{\left(1-|z|^{2}\right)|P(z)|}\right)
\end{aligned}
$$

where the last inequality follows by virtue of Lemma 3 and the triangle inequality. The proof is concluded by applying Lemma 2 with $k=1$ and $\beta=2 \alpha-1$.

Figure 1 shows the transitional curve determined by $R^{\prime}=R_{b}$ for various values of $r$ from 0.3 to 0.6 .

REMARK 2. From Remark $1, R_{1} \leqq R_{b}$ for $b \in[0,1]$. Thus $\alpha A_{1} \leqq R_{b}^{2}$ whenever $\alpha A_{1} \leqq R_{1}^{2}$ or equivalently, when

where

$$
\begin{aligned}
0 & \leqq\left(1+(2 \alpha-1) r^{2}\right)(1-r)-\alpha\left(1-(2 \alpha-1) r^{2}\right)(1+r) \\
& =(1-\alpha) W(r, \alpha)
\end{aligned}
$$

$$
\begin{aligned}
W(r, \alpha) & =1-3 r-3(2 \alpha-1) r^{2}+(2 \alpha-1) r^{3} \geqq 1-3 r-3 r^{2}+r^{3} \\
& =(1-r)\left(1-4 r+r^{2}\right) \geqq 0
\end{aligned}
$$


for each $\alpha \in[0,1)$. Hence (12) is valid for all $\alpha \in[0,1), b \in[0,1]$ and $r \in\left[0,2-3^{1 / 2}\right]$. Thus the transitional curve for $r=2-3^{1 / 2}$ would just touch the upper right-hand corner of the square in Figure 1.

3. The class $\mathscr{P}_{a}^{\prime}(\alpha)$. Let $\mathscr{P}_{a}^{\prime}(\alpha)$ denote the class of functions $F(z)=$ $z+a(1-\alpha) z^{2}+\cdots$ such that $F^{\prime}(z) \in \mathscr{P}_{a}(\alpha)$. Results concerning distortion and regions covered by the class together with other references may be found in [1]. A radius of convexity theorem is also presented in [1] for the class $\mathscr{P}_{a}^{\prime}(\alpha)$; the result, however, is exact only for the case $\alpha=0$. Here we will produce a sharp estimate for all $a \in[0,1]$ and $\alpha \in[0,1)$.

From Nehari [2] it is known that $F(z)$ maps $|z|<r$ onto a convex region if $\operatorname{Re}\left\{1+z F^{\prime \prime}(z) \mid F^{\prime}(z)\right\}>0$ for $|z|<r$.

LemMa 4. If $F(z) \in \mathscr{P}_{a}^{\prime}(\alpha)$, then, for all $\alpha \in[0,1)$ and $a \in[0,1]$,

$$
\operatorname{Re}\left\{1+\frac{z F^{\prime \prime}(z)}{F^{\prime}(z)}\right\}
$$

$$
\geqq \frac{1+4 \alpha a r+\left(6 \alpha-4+4 a^{2} \alpha\right) r^{2}+4(2 \alpha-1) a r^{3}+(2 \alpha-1) r^{4}}{\left(1+2 \alpha a r+(2 \alpha-1) r^{2}\right)\left(1+2 a r+r^{2}\right)}
$$

$$
\geqq \frac{(1-2 \alpha)-A_{1}+2\left(\alpha A_{1}\right)^{1 / 2}}{1-\alpha} \text { if } R_{a} \leqq R^{\prime}
$$

where

$$
R_{a}=\frac{1+2 \alpha a r+(2 \alpha-1) r^{2}}{1+2 a r+r^{2}}, \quad R^{\prime}=\left(\alpha A_{1}\right)^{1 / 2}, \quad r=|z|<1 .
$$

Proof Let $P(z)=F^{\prime}(z)$, then $\operatorname{Re}\left\{1+z F^{\prime \prime}(z) / F^{\prime}(z)\right\}=1+\operatorname{Re}\left\{z P^{\prime}(z) / P(z)\right\}$ and we may apply Theorem 1 .

THEOREM 2. Each $F(z) \in \mathscr{P}_{a}^{\prime}(\alpha)$ maps $|z|<R$ onto a convex region where $R$ is the smallest positive root of the equation

$$
1+4 \alpha a r+\left(6 \alpha-4+4 a^{2} \alpha\right) r^{2}+4(2 \alpha-1) a r^{3}+(2 \alpha-1) r^{4}=0
$$

if $R_{a} \geqq R^{\prime}$, and $R=\left(1+\left(\alpha^{-1}-1\right)^{1 / 2}\right)^{1 / 2}$ if $R_{a} \leqq R^{\prime}, R_{a}$ and $R^{\prime}$ are as in Lemma 4. The result is sharp for each $\alpha \in[0,1)$ and $a \in[0,1]$.

Proof. Apply Lemma 4. For sharpness consider

$$
\begin{aligned}
& F(z)=-(1-2 \alpha) z+(1-\alpha)((1-a) \cdot \log (1+z)-(1+c) \cdot \log (1+z)) \\
& \text { if } R_{a} \geqq R^{\prime} \text {, } \\
& F(z)=-(1-2 \alpha) z+(1-\alpha)((1-c) \cdot \log (1+z)-(1+c) \cdot \log (1-z)) \\
& \text { where } c \text { is determined from } R_{c}=R^{\prime} \text {. } \\
& \text { if } R_{a} \leqq R^{\prime}
\end{aligned}
$$




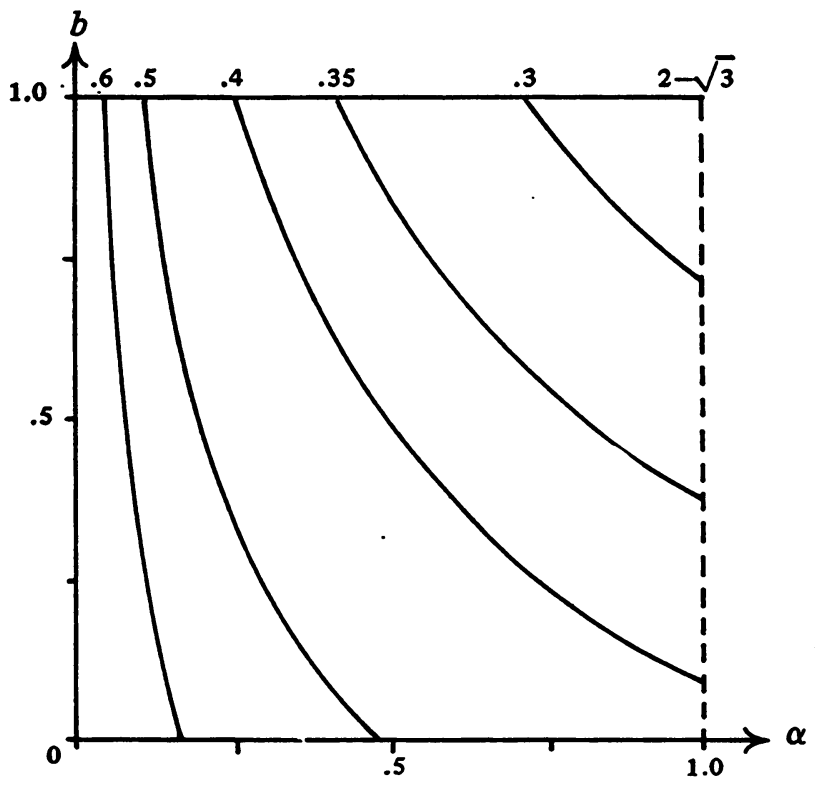

FIGURE 1

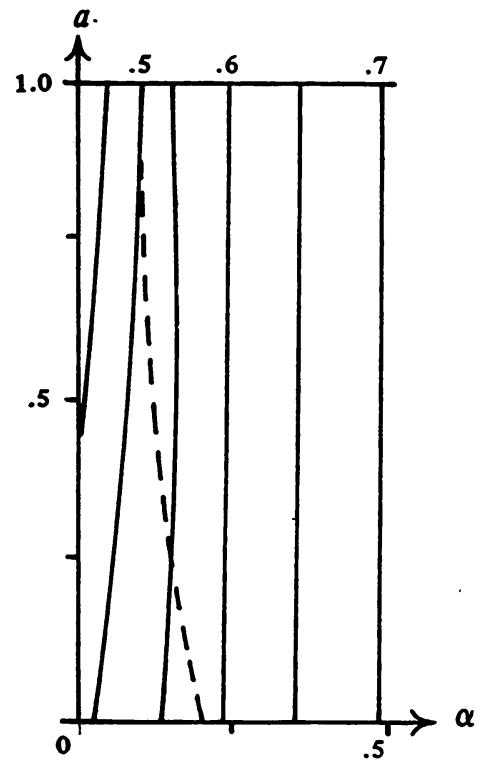

FIGURE 2

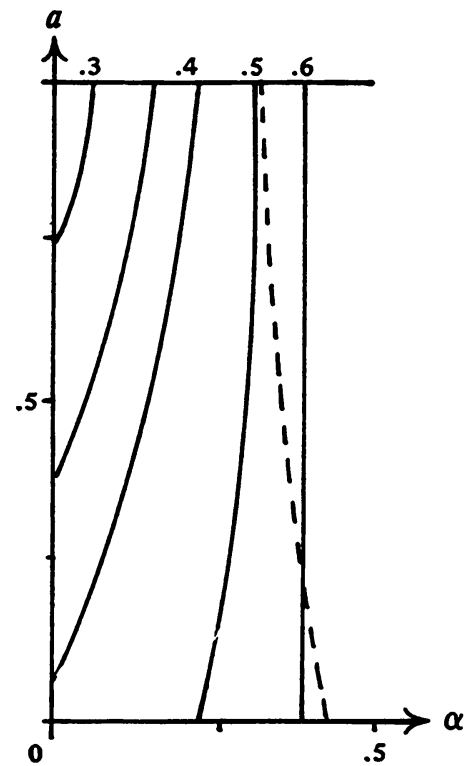

Figure 3 
Figure 2 shows the transitional line $R_{a}=R^{\prime}$ and displays some level curves for various values of the radius of convexity.

4. The class $\mathscr{S}_{a}^{*}(\alpha)$. Let $\mathscr{S}^{*}(\alpha)$ represent the class of functions $f(z)=z+a_{2} z^{2}+\cdots$ which are analytic and for which $\operatorname{Re}\left\{z f^{\prime}(z) \mid f(z)\right\}>\alpha$ for $\alpha \in[0,1)$ and $|z|<1$. A. Schild [3] has shown that $\left|a_{2}\right| \leqq 2(1-\alpha)$ and obtained results on the radius of convexity for a subclass of $\mathscr{S}^{*}(\alpha)$. Singh and Goel [4] obtained the exact radius of convexity for the entire class $\mathscr{S}^{*}(\alpha)$. Define

$$
\mathscr{S}_{a}^{*}(\alpha)=\left\{f(z)=z+2 a(1-\alpha) z^{2}+\cdots: f(z) \in \mathscr{S}^{*}(\alpha)\right\} .
$$

D. Tepper [5] found the exact radius of convexity for $\mathscr{S}_{a}^{*}(0)$. Here we determine a sharp estimate for the entire class $\mathscr{S}_{a}^{*}(\alpha)$.

LEMma 5. If $f(z) \in \mathscr{S}_{a}^{*}(\alpha)$, then, for all $\alpha \in[0,1)$ and $a \in[0,1]$,

$$
\operatorname{Re}\left\{1+\frac{z f^{\prime \prime}(z)}{f^{\prime}(z)}\right\}
$$

$$
\geqq \frac{1+(6 \alpha-2) a r+\left(4 \alpha^{2} a^{2}+8 \alpha-6\right) r^{2}+\left(8 \alpha^{2}-2 \alpha-2\right) a r^{3}+(2 \alpha-1) r^{4}}{\left(1+2 a r+r^{2}\right)\left(1+2 \alpha a r+(2 \alpha-1) r^{2}\right)}
$$

$$
\text { if } R_{a} \geqq R^{\prime}
$$

(17) $\geqq \frac{2\left(\alpha(2-\alpha) A_{1}\right)^{1 / 2}-\alpha-A_{1}}{1-\alpha}$ if $R_{a} \leqq R^{\prime}$

where

$$
R_{a}=\frac{1+2 \alpha a r+(2 \alpha-1) r^{2}}{1+2 a r+r^{2}}, \quad R^{\prime}=\left(\frac{\alpha}{2-\alpha} A_{1}\right)^{1 / 2}, \quad r=|z|<1 .
$$

Proof. Let $P(z)=z f^{\prime}(z) / f(z)$, then $\operatorname{Re}\left\{1+z f^{\prime \prime}(z) / f^{\prime}(z)\right\}=\operatorname{Re}\{P(z)\}+$ $\operatorname{Re}\left\{z P^{\prime}(z) / P(z)\right\}$. Now apply Lemma 2 with $\beta=2 \alpha-1$ and $k=3-2 \alpha$.

THEOREM 3. Each $f(z) \in \mathscr{S}_{a}^{*}(\alpha)$ maps $|z|<R$ onto a convex region where $R$ is the smallest positive root of the equation

$$
\begin{aligned}
1+(6 \alpha-2) a r+\left(4 \alpha^{2} a^{2}+8 \alpha\right. & -6) r^{2} \\
& +\left(8 \alpha^{2}-2 \alpha-2\right) a r^{3}+(2 \alpha-1)^{2} r^{4}=0
\end{aligned}
$$

if $R_{a} \geqq R^{\prime}$ and $R=\left((5 \alpha-1) /\left(4 \alpha^{2}-\alpha+1+4 \alpha\left(\alpha^{2}-3 \alpha+2\right)^{1 / 2}\right)\right)^{1 / 2}$ if $R_{a} \leqq R^{\prime}$ where $R_{a}$ and $R^{\prime}$ are as in Lemma 5. The result is sharp for each $\alpha \in[0,1)$ and $a \in[0,1]$.

Proof. Apply Lemma 5. For sharpness, let

$$
f(z)=z /\left(1-2 a z+z^{2}\right)^{1-\alpha}, \quad \text { if } R_{a} \geqq R^{\prime},
$$


and

$$
f(z)=z /\left(1-2 c z+z^{2}\right)^{1-\alpha}, \quad \text { if } R_{a} \leqq R^{\prime},
$$

where $c$ is determined from $R_{c}=R^{\prime}$.

Figure 3 gives some level curves and the transitional curve for the class $\mathscr{S}_{a}^{*}(\alpha)$.

REMARK 3. By setting $a=0$ in Theorems 2 and 3 we could obtain sharp results on odd functions in the two classes $\mathscr{P}^{\prime}(\alpha)$ and $\mathscr{S}^{*}(\alpha)$ for $\alpha \in[0,1)$.

\section{REFERENCES}

1. C. P. McCarty, Functions with real part greater than $\alpha$, Proc. Amer. Math. Soc. 35 (1972), 211-216.

2. Z. Nehari, Conformal mapping, McGraw-Hill, New York, 1952. MR 13, 640.

3. A. Schild, On starlike functions of order $\alpha$, Amer. J. Math. 87 (1965), 65-70. MR 30 \#4929.

4. V. Singh and R. M. Goel, On radii of convexity and starlikeness of some classes of functions, J. Math. Soc. Japan 23 (1971), 323-339. MR 43 \#7617.

5. D. E. Tepper, On the radius of convexity and boundarydistortion of schlicht functions, Trans. Amer. Math. Soc. 150 (1970), 519-528. MR 42 \#3268.

Department of Mathematics, la Salle College, Philadelphia, Pennsylvania 19141 